\title{
Anacamptis $x$ rayyana nothosp. nov. (Orchidaceae), un nuevo híbrido para el sur de la Península Ibérica
}

\author{
Estrella Robles-Domínguez ${ }^{1}$, Damián Quintana-Pozo ${ }^{2}$ y Manuel Becerra-Parra ${ }^{1}$ \\ ${ }^{1}$ Bda. San Miguel, 1. 29370 Benaoján (Málaga) \\ 2 Bda. Manuel Ortiz, bloque 1, 4 A. Archidona (Málaga)
}

\author{
Correspondencia \\ M. Becerra-Parra \\ e-mail: manuel.becerra.parra79@gmail.com \\ Recibido: 25 noviembre 2019 \\ Aceptado: 26 febrero 2020 \\ Publicado on-line: 20 abril 2020 \\ Editado por: Marta Recio Criado
}

\begin{abstract}
Resumen
Se propone un nuevo taxón de origen híbrido entre Anacamptis laxiflora (Lam.) R.M. Bateman, Pridgeon \& M.W. Chase y Anacamptis champagneuxil (Barnéoud) R.M. Bateman, Pridgeon \& M.W. Chase, localizado en la provincia de Málaga. Se describe morfológicamente y se comentan las diferencias con los parentales y con Anacamptis $x$ alata (Fleury) H. Kretzschmar, Eccarius \& H. Dietr., nototaxon entre Anacamptis laxiflora y Anacamptis morio (L.) R.M. Bateman, Pridgeon \& M.W. Chase, otro de los taxones que comprende la subsección Moriones en la Península Ibérica.
\end{abstract}

Palabras clave: Anacamptis, híbrido, taxonomía, Málaga, España.

\begin{abstract}
Anacamptis $x$ rayyana nothosp. nov. (Orchidaceae), a new hybrid for the south of the Iberian Peninsula.

A new hybrid taxa between Anacamptis laxiflora (Lam.) R.M. Bateman, Pridgeon \& M.W. Chase and Anacamptis champagneuxii (Barnéoud) R.M. Bateman, Pridgeon \& M.W. Chase located in the province of Malaga is suggested. It is morphologically described and the differences with its parents and with Anacamptisx alata (Fleury) H. Kretzschmar, Eccarius \& H. Dietr. are explained. Anacamptis $x$ alata (Fleury) H. Kretzschmar, Eccarius \& H. Dietr. is an hybrid name between Anacamptis laxiflora and Anacamptis morio (L.) R.M. Bateman, Pridgeon \& M.W. Chase, taxa that also belongs to subsection Moriones in the Iberian Peninsula.
\end{abstract}

Key words. Anacamptis, hybrid, taxonomy, Malaga, Spain.
El género Orchis ha incluido tradicionalmente un conjunto de especies con morfologías muy variadas pero que se caracterizaban por sus tubérculos indivisos, brácteas florales membranáceas y flores con espolón (Aedo, 2005). Sin embargo, a finales del pasado siglo el desarrollo de las técnicas moleculares, basadas en los marcadores ITS del ADN ribosómico, motivaron la inclusión de los distintos taxones que incluía la antigua sección Platycheilae del género Orchis, dentro de la que se incluían los grupos de Orchis morio L. y Orchis laxiflora Lam., en el género Anacamptis, hasta entonces monoespecífico (Bateman et al., 1997).

La sección Laxiflorae (grupo de Anacamptis laxiflora), que incluye en el ámbito peninsular dos especies, A. laxiflora y A. palustris (Jacq.) R.M. Bateman, Pridgeon \& M.W. Chase, se caracteriza por sus sépalos laterales dirigidos hacia atrás, de erectos a patentes, y sépalo central connivente con los pétalos formando una gálea, además, de su ecología (suelos muy húmedos o encharcados, incluso en ambiente lacuste).
Por su parte, la sección Moriones (grupo de Anacamptis morio) estaría representada en la Península lbérica por dos taxones, Anacamptis morio y Anacamptis champagneuxii, a la que se sumaría un tercero, Anacamptis picta (Loisel) R.M. Bateman, Pridgeon \& M.W. Chase, cuyo estatus taxonómico aún no está resuelto en nuestro ámbito de estudio. Comprende a especies con flores de labelo profundamente dividido con el lóbulo central subigual o más corto que los laterales y sépalos conniventes en una gálea que junto a los pétalos cubren el ginostemo.

Dentro de la familia Orchidaceae la hibridación es un fenómeno natural relativamente frecuente (Kretzschmar et al., 2007) y el género Anacamptis no es una excepción. Para el Paleártico Occidental hay descritos un total de 55 nothotaxones (Kretzschmar et al., 2007).

Durante nuestros trabajos de campo localizamos unas plantas en el seno de una población en la que crecían conjuntamente Anacamptis champagneuxii y Anacamptis laxiflora que llamaron nuestra atención. 
Tras recolectar material para su estudio apreciamos que éstas presentaban caracteres morfológicos intermedios entre ambos taxones, llegando a la conclusión de que estábamos ante un híbrido aún no descrito (Kretzschmar et al., 2007) (Fig. 1).

Hemos depositado el tipo en el herbario MGC. El resto del material estudiado de Anacamptis $x$ alata (Fleury) H. Kretzschmar, Eccarius \& H. Dietrich se encuentra depositado en el herbario PAMP.

Anacamptis x rayyana E. Robles, D. Quintana \& M. Becerra noth. nov.

Typus: España, Málaga, Colmenar, Sierra Prieta. 30STF79. Praderas higrófilas, 1.200 m. 11-V-2019. Leg. D. Quintana, M. Cabrera, J. A. Díaz Rodriguez, J. A. Díaz Romera \& J. Cebrián. Det. M. Becerra \& E. Robles. MGC 92648.

Diagnosis. Difiere de Anacamptis laxiflora por su labelo no plegado y nada o sutilmente geniculado; contrariamente a Anacamptis champagneuxii no presenta los sépalos laterales dirigidos hacia delante y conniventes con el sépalo central formando una gálea, sino que son suberectos; franja central del labelo blanca, con una a cinco máculas longitudinales, a veces desdibujadas. La relación longitud espolón/longitud labelo es notablemente mayor que en los parentales (Fig. 2).

Diagnosis. It differs from Anacamptis laxiflora by its unfolded lip not or slightly geniculate and from Anacamptis champagneuxii because this hybrid does not show lateral sepals forward and connivent with the central sepal forming a galea, but rather they are sub-straight; with a central white strip in the lip and one to five longitudinal macules, sometimes blurred. The ratio between the spur length and lip length is markedly greater than in parental ones.

Descripción: Plantas hasta de $30 \mathrm{~cm}$. Hojas dispuestas en roseta basal, no maculadas. Inflorescencia laxa, con 6-9 flores, eje de color púrpura. Brácteas 14-20 × $4 \mathrm{~mm}$, lanceoladas, de tonalidad rosado-purpúrea, con nerviación verdosa; la inferior de igual longitud que el ovario o algo más corta. Flores de color rosa-violáceo a rosa-púrpura. Sépalos ovado-lanceolados, asimétricos y obtusos; los laterales suberectos, parcialmente dirigidos hacia atrás; el central dirigido hacia delante. Pétalos laterales 7-8 x $2 \mathrm{~mm}$, de menor tamaño que los sépalos, rectangulares, obtuoso, conniventes, cubriendo el ginostemo. Labelo 11-14 x 5-7 mm, de contorno reniforme, subentero 0 sutilmente trilobulado, levemente convexo y nada o ligeramente geniculado, con la franja central blanquecina, recorrida en la mayor parte de las plantas observadas por entre una y cinco máculas longitudinales concoloras al labelo y en ocasiones desdibujadas. Espolón 9-12 mm, algo ensanchado en su extremo distal, curvado hacia arriba, con el ápice bífico a subbífido; relación longitud espolón/longitud labelo = 1,8-1,71.

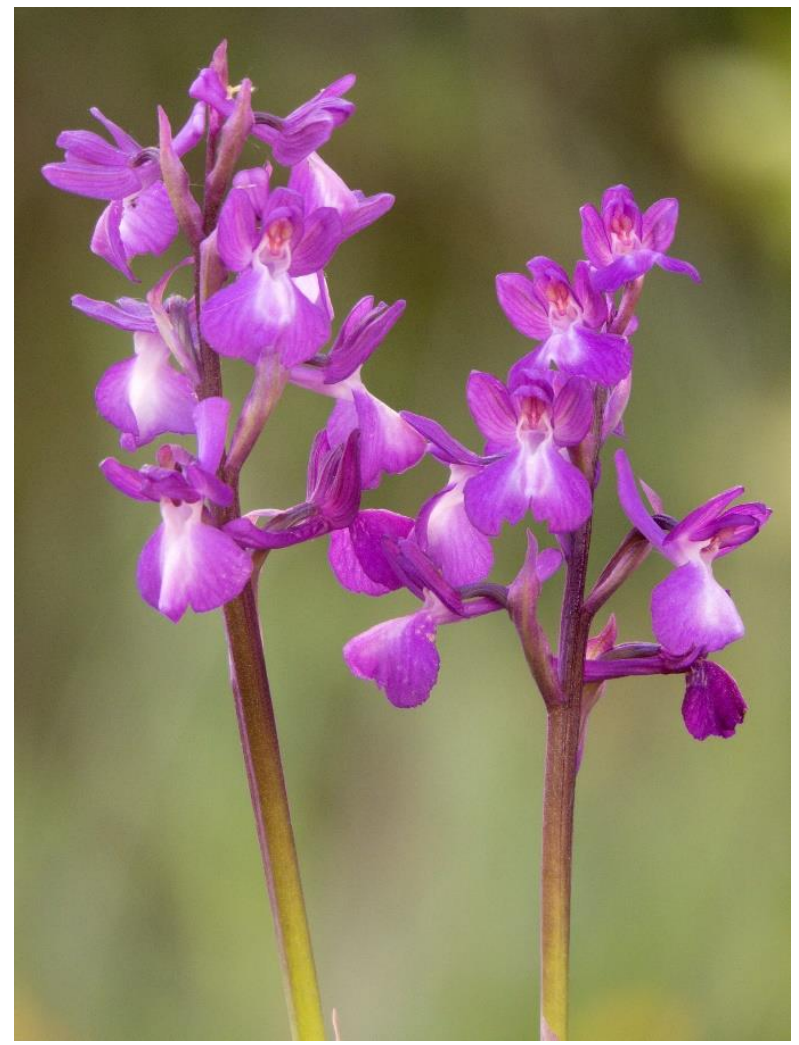

Figura 1. Anacamptis x rayyana.

Figure 1. Anacamptis x rayyana.

Etimología: El epíteto rayyana proviene de la cora andalusí de Rayya, cuya capital fue Medina Arxiduna y abarcó buena parte de la actual provincia de Málaga.

Distribución y hábitat: Tan sólo hemos localizado una población, integrada por seis individuos, en el extremo occidental del Arco Calizo Central Malagueño. Ocupa praderas graminoides higrófilas y verticícolas supra-mesomediterráneas de la asociación Elymo repentis-Phalaridetum caerulescentis Pérez Latorre, Galán \& Cabezudo in Pérez Latorre, P. Navas, D. Navas, Y. Gil \& Cabezudo, las cuales aparecen en los suelos arcillosos que se desarrollan en depresiones kársticas y que mantienen la humedad hasta finales de primavera (Cabezudo et al., 2016). Algunas de las especies acompañantes son Xiphion vulgare Mill., Phlomis herba-venti L., Carduncellus caeruleus (L.) C. Prel., Phalaris caerulescens Desf. y Hordeum bulbosum L.

Fenología: Florece durante la primera quincena de mayo.

En la localidad donde se ha recolectado este nuevo nototaxon, las plantas hibridógenas conviven con Anacamptis laxiflora y Anacamptis champagneuxii, presentando éstas caracteres 
morfológicos intermedios entre ambos parentales, como los sépalos laterales suberectos y levemente dirigidos hacia atrás, no conniventes con el sépalo central; labelo no plegado ni geniculado o si acaso sutilmente en algunas flores, subentero o un poco trilobulado, con el lóbulo central que apenas sobresale; y franja central del labelo blanquecina, con una o varias máculas longitudinales. Comparte, sin embargo, otros aspectos morfológicos con sus progenitores como el ápice bífido del espolón, siendo éste de una longitud intermedia entre ambos parentales. Aun así, se observa una cierta variabilidad en la morfología floral, sobre todo en lo referente al labelo, siendo más similar ésta a la de Anacamptis laxiflora (Fig. 2).

Salvo el caso del híbrido entre Anacamptis morio y Anacamptis laxiflora, Anacamptis $x$ alata (Fleury) H. Kretzschmar, Eccarius \& H. Dietr., no se ha descrito ningún otro notaxón entre Anacamptis laxiflora y las otras especies que integran el grupo de Anacamptis morio, a pesar de que parece que en el sur de Francia los híbridos entre Anacamptis laxiflora y Anacamptis champagneuxii no deben ser raros (Kretzschmar et al., 2007).

En la bibliografía consultada no hemos encontrado citas anteriores de ninguno de los nototaxones entreAnacamptis laxiflora y Anacamptis gr. morio para la Península Ibérica (Benito Ayuso, 2017; Aedo, 2005). Tan sólo existe un pliego en el
Herbario del Real Jardín Botánico de Madrid (MA23915), determinado por S. Rivas Goday como Orchis laxiflora, donde uno de los ejemplares parece corresponderse con un posible híbrido entre Anacamptis laxiflora y Anacamptis champagneuxii o Anacamptis morio (Benito Ayuso, 2017).

Hemos localizado en Navarra, en un humedal de la Cuenca de Pamplona, Anacamptis $x$ alata (Fig. 3). Éste presenta una morfología floral que la separa notoriamente de Anacamptis x rayyana, destacando como caracteres diferenciadores las numerosas máculas, bien definidas, presentes en la franja central del labelo, muy representativas de Anacamptis morio; el lóbulo central del labelo, que en este caso si está bien marcado; y su espolón recto, muy largo en relación al labelo y con el ápice bífido, caracter, este último, ausente en Anacamptis morio pero sí presente en Anacamptis laxiflora.

\section{Material estudiado:}

Anacamptis $x$ alata (Fleury) H. Kretzschmar, Eccarius \& H. Dietr.

Navarra. Cendea de Iza, concejo de Iza. Proximidades de la balsa de Iza. 30TXN0343. Herbazales sobre suelos encharcados. Arcillas. 17V-2019. Leg. E. Robles \& M. Becerra. Det. E. Robles. PAMP 56186.

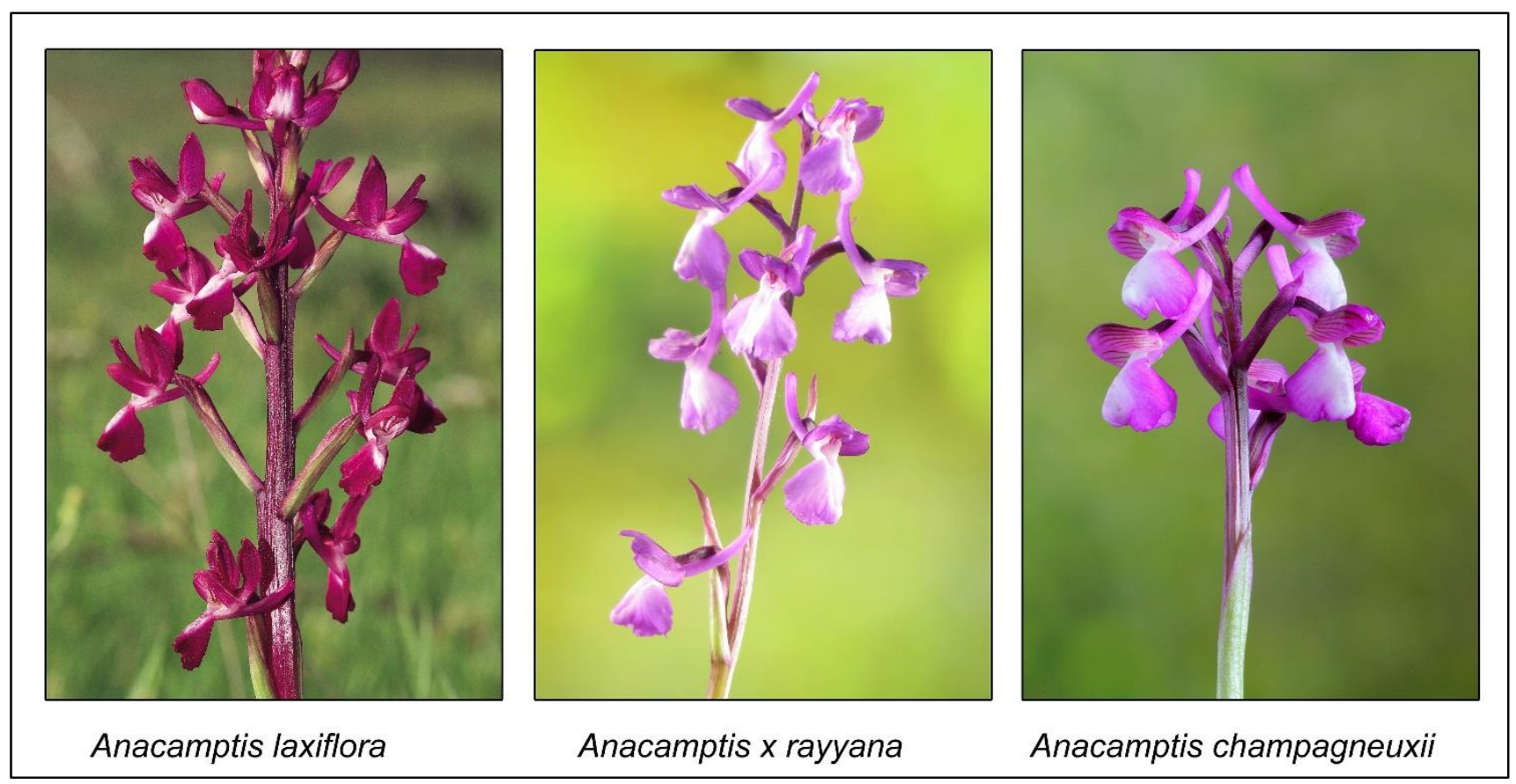

Figura 2. Comparativa entre Anacamptis x rayyana y sus parentales.

Figure 2. Comparison between Anacamptis x rayyana and its parents. 


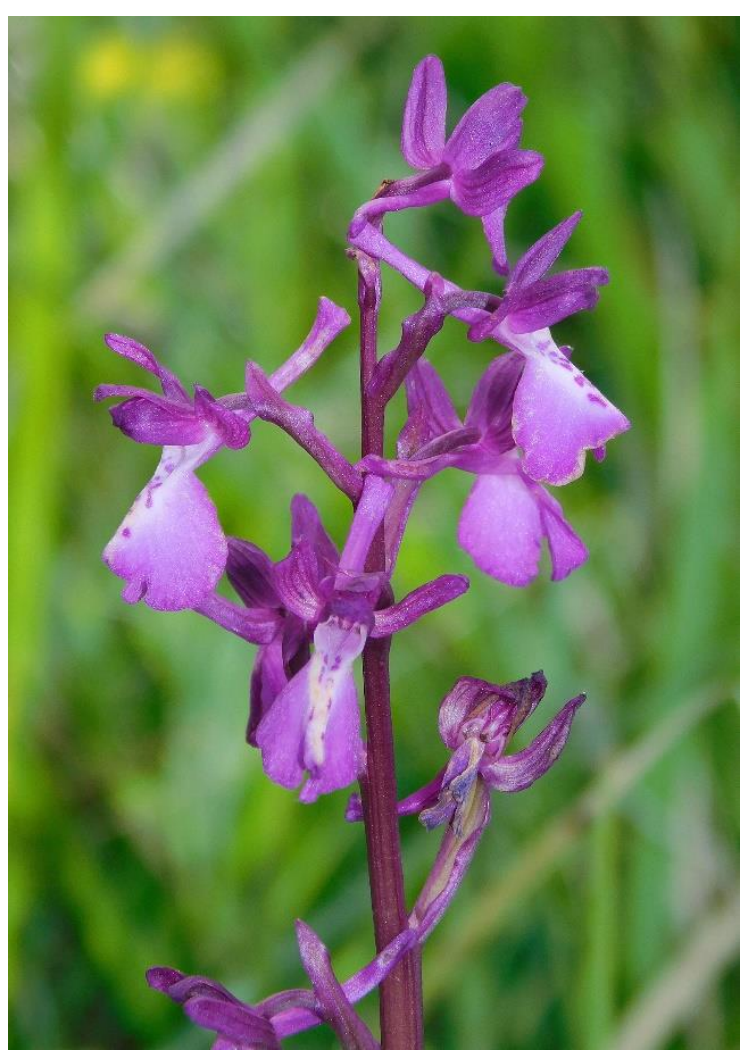

Figura 3. Anacamptis $x$ alata.

Figure 3. Anacamptis $x$ alata

\section{Agradecimientos}

A Javier Benito Ayuso por la lectura crítica del texto $y$ sus enriquecedoras aportaciones $y$ sugerencias.

A Miguel Cabrera, Juan Cebrián, José Antonio Díaz Romera, José Antonio Díaz Rodríguez y Joaquín Ramírez, quienes nos han acompañado en nuestras salidas para estudiar la orquideoflora de la provincia de Málaga.
A los responsables de los herbarios MGC y PAMP por las facilidades para depositar el material de herbario.

A Alberto Martínez por su ayuda en la traducción del resumen.

\section{Bibliografía}

Aedo, C. (2005) Orchis L. En C. Aedo \& A. Herrero (eds.) Flora Iberica vol. XXI SmilaceaeOrchidaceae (pp. 114-146). Madrid, España: Real Jardín Botánico, CSIC.

Algarra, J. \& Blanca, G. (2009). Orchidacea L. En G. Blanca, B. Cabezudo, M. Cueto, C. Fernández López \& C. Morales Torres (eds.). Flora Vascular de Andalucía Oriental. Sevilla, España: Consejería de Medio Ambiente, Junta de Andalucía.

Benito Ayuso, J. (2017). Estudio de las orquídeas silvestres del Sistema lbérico (tesis doctoral). Universidad de Valencia, Valencia, España.

Bateman, R.M., Pridgeon, A.M. \& Chase, M.W. (1997). Phylogenitcs of subtribe Orchidinae (Orchidoideae, Orchidaceae) based on nuclear ITS sequences 2. Infrageneric relationships and taxonomic revision to achieve monophyly of Orchis sensu sctricto. Lindleyana, 12, 113-141.

Cabezudo, B., Casimiro Soriguer-Solanas, F., García-Sánchez, J. \& Pérez Latorre, A.V. (2016). Flora y vegetación de la Zona de Especial Conservación (ZEC) Sierra de Camarolos (Málaga, España). Acta Botanica Malacitana, 41,163-246.

doi: $10.24310 /$ Actabotanicaabm.v41io.2448

Kretzschmar, H., Eccarius, W. \& Dietrich, H. (2007). The Orchid Genera. Anacamptis, Orchis, Neotinea. Phylogeny, Taxonomy, Morphology, Biology, Distribution, Ecology and Hybridisation. Albersdorf, Alemania: EchinoMedia. 